\title{
Weightbearing CBCT, MDCT, and 2D imaging dosimetry of the foot and ankle
}

\author{
John B. Ludlow ${ }^{1}$, Marija I vanovic ${ }^{2}$ \\ 1. Department of Diagnostic Sciences, University of North Carolina School of Dentistry, Chapel Hill, North Carolina, USA. \\ 2. Department of Radiology, University of North Carolina Hospitals, Chapel Hill, North Carolina, USA.
}

Correspondence: John B. Ludlow, Professor. Address: Koury Oral Health Sciences, Room 5411-K 385 South Columbia StreetChapel Hill NC 27599-7455, USA. Email: jbl@ad.unc.edu

Received: February 7, 2014

Accepted: February 19, $2014 \quad$ Online Published: March 5, 2014

DOI : $10.5430 /$ ijdi.v1n2p1

URL: http://dx.doi.org/10.5430/ijdi.v1n2p1

\begin{abstract}
Aims: This study compares effective doses associated with 2D lateral, oblique, and AP radiographs with 3D MDCT and Cone Beam CT images of the foot and ankle.

Methods: An anthropomorphic phantom of the foot and ankle was constructed from an adult human skeleton and soft tissue equivalent material. Optical stimulated dosimeters were placed at 21 locations within and on tissues and anatomy of interest. Effective dose was calculated following 2007 ICRP recommendations. Three projections were exposed to simulate conventional 2D imaging. Standard and optimized dose MDCT scans were exposed to simulate typical CT options. Ten, 20 , and $30 \mathrm{~cm}$ fields of view, 100 and $120 \mathrm{kVp}$ and 4.5 and $6.8 \mathrm{mAs}$ exposures were tested using a PedCAT ${ }^{\circledR}$ CBCT device designed for weightbearing imaging. Dose was calculated for an adult as well as for a 5 and 10-year-old child to assess the impact of age on risk estimation.

Results: Standard adult effective doses for single foot imaging were $0.6 \mu \mathrm{Sv}$ for $2 \mathrm{D}, 3.8 \mu \mathrm{Sv}$ for $\mathrm{CBCT}$, and $25 \mu \mathrm{Sv}$ for MDCT $(p=0.0013)$. Dose differed significantly with age $(p=0.0185)$. For a 5 -year-old, doses rise to $0.8 \mu \mathrm{Sv}$ for $2 \mathrm{D}$, $18 \mu \mathrm{Sv}$ for CBCT, and $200 \mu \mathrm{Sv}$ for MDCT. Small and medium CBCT fields produced adult doses of $2.3 \mathrm{mSv}$ and $0.9 \mathrm{mSv}$ respectively.

Conclusions: The effective dose for small FOV CBCT or conventional 2D series examinations is comparable to a few hours of equivalent background dose. Such doses are negligible; therefore, the dose of radiation should not be a concern when considering the use of CBCT imaging for foot/ankle examination.
\end{abstract}

\section{Keywords}

Radiation Dosimetry, Radiography, Cone Beam CT, Spiral CT scan, Foot bones, Ankle bones

\section{I ntroduction}

Bony pathologies resulting from trauma and osteoarthritic changes in the foot and ankle, while commonplace, are often difficult to visualize with "plain film" transmission radiography ${ }^{[1,2]}$. Multiple views are required and often are unsatisfactory due to superimposition of multiple adjacent bones that form the complex skeletal structure of this anatomy ${ }^{[3]}$. Multi-detector Computed Tomography (MDCT) is an good diagnostic alternative and permits isolation and 
visualization of separate bony structures ${ }^{[4,5]}$; however, this examination is expensive, limited in availability to hospitals and large radiology practices, and is associated with significantly increased x-ray dose in relation to plain views.

Cone Beam CT (CBCT) represents a less expensive alternative to MDCT that has found increasing application in radiation therapy planning / monitoring and maxillofacial imaging over the last decade. While limited in its ability to visualize soft tissue structures, this modality is perhaps ideally suited to providing definitive imaging of the osseous components of the foot and ankle.

It has been estimated that from $1.5 \%$ to $2 \%$ of all US cancers may be attributed to computed tomography (CT) studies alone ${ }^{[6]}$. Medical CT scan doses vary widely depending on the anatomic region and purpose of the scan ${ }^{[7]}$. Dose is especially important when considering the adolescent and pediatric populations in whom cellular growth and organ development is associated with increased radiosensitivity of tissues. In conjunction with a longer life expectancy in which cancer can develop, children may be two times to five times more sensitive to radiation carcinogenesis as mature adults ${ }^{[7,8]}$. In its applications in head and neck imaging, CBCT has been suggested as a low dose alternative to MDCT ${ }^{[9]}$. While the majority of $\mathrm{CBCT}$ examinations have been reported to impart a much lower dose, CBCT units from different manufacturers have been shown to vary in patient dose 10-fold for an equivalent field of view (FOV), with some units roughly equivalent in dose to optimized CT scans ${ }^{[10]}$. Although experimental ${ }^{[11]}$, and commercial ${ }^{[12]}$, CBCT units have been developed for extremity imaging, the PedCAT ${ }^{\circledR}$ (CurveBeam, Warrington, PA) is the first application of CBCT dedicated to weightbearing imaging of the foot and ankle.

Because dose is a general concern in diagnostic imaging and cost and risks of imaging must be weighed against anticipated benefits of improved diagnosis and resulting management, it is important to define the risk of any radiographic examination. We are unaware of contemporary reports of dose and risk from CT imaging of the foot and ankle. Therefore, the aim of this research is to acquire and compare effective dose data for foot / ankle scanning using 3D CBCT and MDCT methods as well as $2 \mathrm{D}$ transmission radiography imaging.

\section{Methods}

An anthropomorphic phantom of the foot and ankle was constructed permitting localization of dosimeters within and on tissues /anatomy of interest (see Figure 1). The phantom was constructed using the lower leg, ankle, and foot bones of a human skeleton, which was embedded in a radiologically soft-tissue-equivalent material approximating the form of the lower adult extremity (The Phantom Laboratory, Salem, NY). The foot and ankle portion of the phantom was fabricated in $25 \mathrm{~mm}$ horizontally sliced layers allowing separation and specific localization of dosimeters within each layer. Slots were drilled in the surface of the layers to accommodate placement of dosimeters in tissues used in the 2007 ICRP calculation of effective dose (see Table 1). Additional dosimeters were placed in surface areas to measure skin dose. Twenty-one dosimeter locations were utilized (see Figure 2).
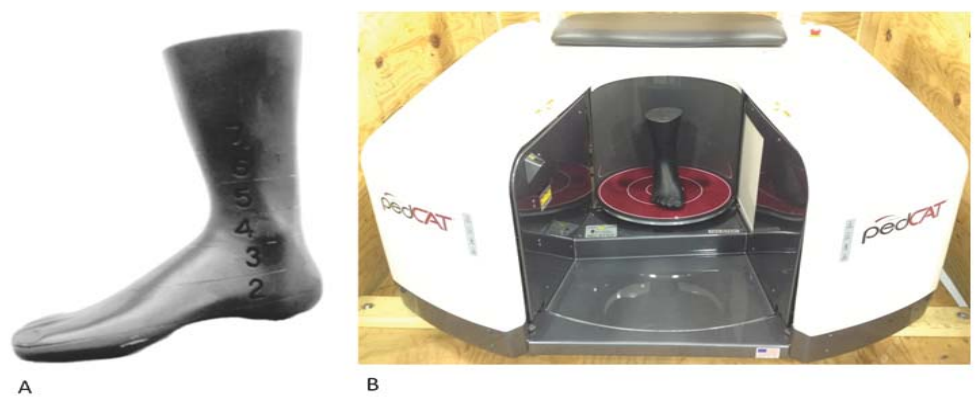

Figure 1. A) Assembled foot phantom and B) Phantom positioned in CBCT unit 
Table 1. Tissue weighting factors for calculation of Effective Dose - ICRP 2007 recommendations.

\begin{tabular}{ll}
\hline Tissue & $\boldsymbol{w}_{\mathbf{T}}$ \\
\hline Bone marrow & 0.12 \\
Breast & 0.12 \\
Colon & 0.12 \\
Lung & 0.12 \\
Stomach & 0.12 \\
Bladder & 0.04 \\
Esophagus & 0.04 \\
Gonads & 0.08 \\
Liver & 0.04 \\
Thyroid & 0.04 \\
Bone surface & 0.01 \\
Brain & 0.01 \\
Salivary glands & 0.01 \\
Skin & 0.01 \\
Remainder Tissues & $0.12 \dagger$ \\
\hline
\end{tabular}

-Adrenals, Extrathoracic region, Gall bladder, Heart, Kidneys, Lymphatic nodes, Muscle, Oral Mucosa, Pancreas, Prostate, Small Intestine, Spleen, Thymus, and Uterus/cervix. (Bolded text represents directly irradiated tissues used for calculation of foot and ankle dose)

\begin{tabular}{|c|c|}
\hline \multicolumn{2}{|l|}{ Dosimeter Location (Level) } \\
\hline \multicolumn{2}{|l|}{ Medial surface (6) } \\
\hline \multicolumn{2}{|l|}{ Skin anterior surface (5) } \\
\hline \multicolumn{2}{|l|}{ Skin posterior surface $(5)$} \\
\hline \multicolumn{2}{|l|}{ Soft tissues between tibia fibula (5) } \\
\hline \multicolumn{2}{|l|}{ Fibula (5) } \\
\hline Tibia (5) & 5 \\
\hline \multirow{2}{*}{\multicolumn{2}{|c|}{$\begin{array}{l}\text { Skin posterior surface (4) } \\
\text { Skin medial surface ( } 4 \text { ) }\end{array}$}} \\
\hline & \\
\hline \multicolumn{2}{|c|}{ Muscle/ soft tissue posterior to tibia fibula (4) } \\
\hline \multicolumn{2}{|c|}{ Lateral skin surface (3) } \\
\hline \multicolumn{2}{|l|}{ Talus (3) } \\
\hline \multirow{2}{*}{\multicolumn{2}{|c|}{$\begin{array}{l}\text { Calcaneus (2) } \\
\text { Medial skin surface (2) }\end{array}$}} \\
\hline \multirow{2}{*}{\multicolumn{2}{|c|}{ Upper skin surface (2) }} \\
\hline & \\
\hline \multicolumn{2}{|l|}{ Cuneiform (2) } \\
\hline \multicolumn{2}{|l|}{ Plantar skin surface (1) } \\
\hline \multicolumn{2}{|r|}{ Dosimeter locations of like color are averaged to compute } \\
\hline \multicolumn{2}{|r|}{ skin } \\
\hline Big toe (1) & \\
\hline
\end{tabular}

Figure 2. Dosimeter locations in foot /ankle phantom by (level)

CBCT dosimetry was performed on a production CBCT unit (PedCAT, CurveBeam Inc., Warrington, PA) (see Figure1). This unit utilizes an amorphous silicon flat panel detector providing $0.37 \mathrm{~mm}$ voxel sizes in a $20 \mathrm{~cm} \mathrm{high}(\mathrm{H}) \mathrm{by} 35 \mathrm{~cm}$ wide (W) large field of view (FOV) or $20 \mathrm{~cm} \mathrm{H} \times 20 \mathrm{~cm} \mathrm{~W}$ medium FOV or $10 \mathrm{~cm} \mathrm{H} \times 10 \mathrm{~cm}$ W small FOV volume options. The unit operates at 100 to $120 \mathrm{kVp}$ and 3 to $5 \mathrm{~mA}$ producing a single foot scan in 19 seconds. Sixteen warm up frames and 361 data frames are acquired during the scanning sequence. A pulsed exposure of 12 msec for standard scans and $18 \mathrm{msec}$ for grid filtered scans is utilized producing scan exposures of 4.5 to $6.8 \mathrm{mAs}$.

MDCT dosimetry was acquired using a Siemens Somatom Definition AS (Siemens Medical Solutions USA, Malvern, PA) using standard and automatic exposure control (CARE Dose) foot imaging protocols. The unit was operated at $120 \mathrm{kVp}$, $55 \mathrm{mAs}$ and pitch of 0.55 (effective mAs set to 100) while acquiring a $20 \mathrm{~cm}$ length extending from the base of the foot. With CARE Dose on, the mAs was reduced, resulting in average effective mAs of 89 (average mAs of 48.95). Both scans 
were acquired using $38.4 \mathrm{~mm}$ longitudinal beam dimension $(128 \mathrm{row} * 0.6 \mathrm{~mm}$ row height detector configuration with flying focal spot).

Conventional 2D radiographic imaging dosimetry was produced with Siemens x-ray tube Optiptop 150/40/80HC. Exposures were made at suggested medium adult settings of $60 \mathrm{kVp}$ with $3.6 \mathrm{mAs}$ for AP and Oblique views and $4.5 \mathrm{mAs}$ for the lateral view. Sample images from each modality are seen in Figure 3.

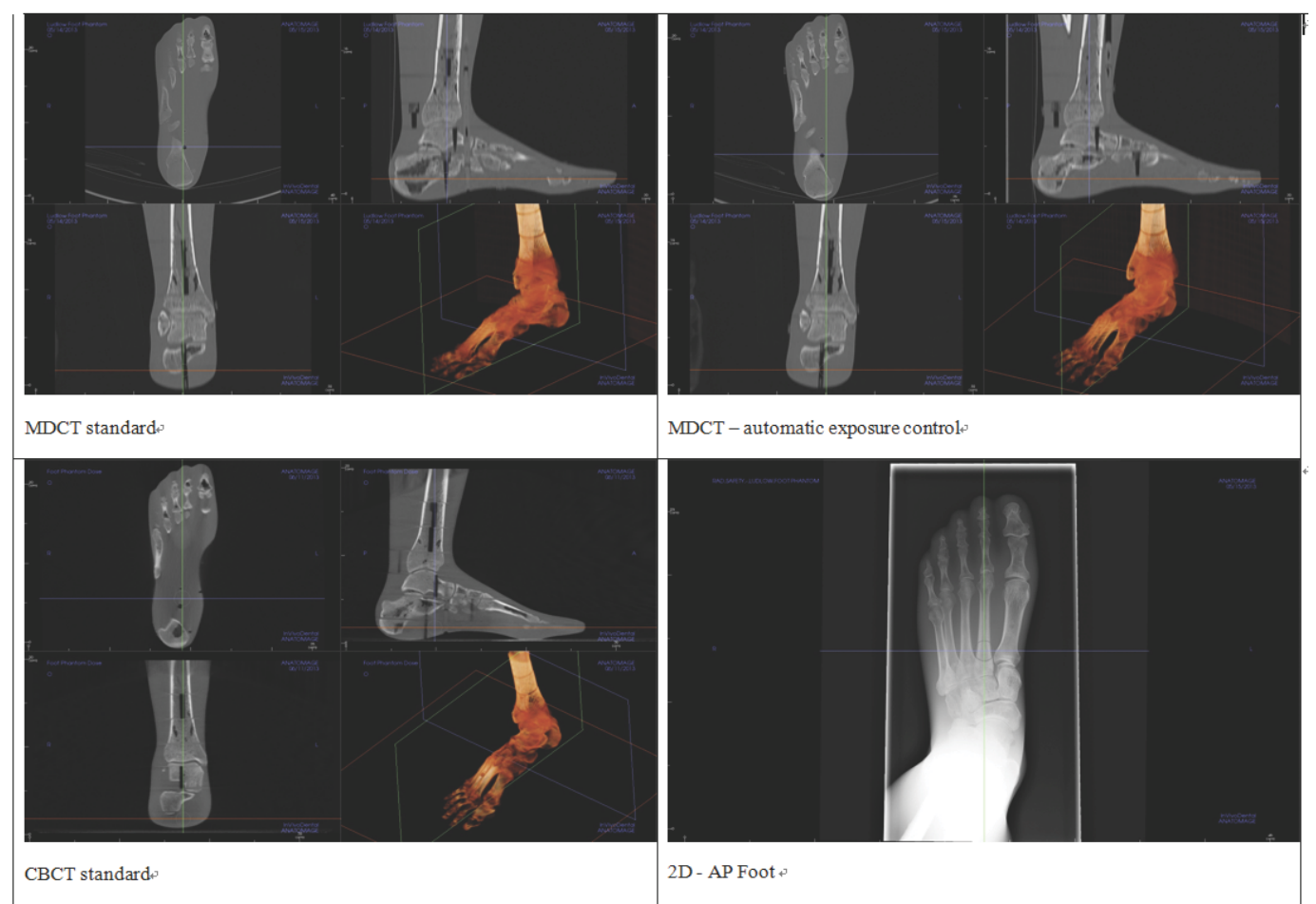

Figure 3. Foot phantom imaging examples.

Optically stimulated luminescent dosimeters (OSL) (Nanodot, Landauer, Inc., Glenwood, IL) were used for dosimetry for this project. NanoDots are plastic disks infused with aluminum oxide doped with carbon $\left(\mathrm{Al}_{2} \mathrm{O}_{3}: \mathrm{C}\right)$. The trace amounts of Carbon in the $\mathrm{Al}_{2} \mathrm{O}_{3}$ crystal lattice create imperfections that act as traps ( $\mathrm{F}$ centers) for electrons or holes. After exposure to ionizing radiation, free electrons and holes are generated and trapped at the $\mathrm{F}$ centers in proportion to the amount of energy in the exposure. Energy captured by the F centers is reemitted as light when electrons or holes recombine. This occurs when the crystal is optically stimulated with a controlled exposure of $540 \mathrm{~nm}$ light from a light emitting diode. The energy released from $F$ centers can be distinguished from the stimulating light because it is emitted in the form of $420 \mathrm{~nm}$ photons. The intensity of the emitted luminescence depends on the dose absorbed by the OSLD and the intensity of the stimulation light. This intensity is proportional to the stored dose and is recorded by a photomultiplier tube that incorporates a filter that screens out photons from the stimulating light source. Each dosimeter is encased in a light-tight plastic holder measuring approximately $1 \mathrm{~mm} \times 10 \mathrm{~mm} \times 10 \mathrm{~mm}$. This case prevents loss of energy through stimulation by ambient light. Dosimeters used in this study were read with a portable reader (MicroStar, Landauer, Inc., Glenwood, IL). The reader was calibrated before use. Following calibration, photon counts from dosimeters were recorded with an accuracy of $\sim+/-2 \%$. Photon counts were converted to dose using an energy specific conversion factor (ECF).

Multiple exposures were utilized for some dosimeter runs to provide a more reliable measure of radiation in the dosimeters. OSLD doses recorded by the reader were divided by the number of scans (or projections for plain film techniques) to determine the 'exposure per examination' for each dosimeter. Dosimeter values were adjusted for energy response of the OSL material using polynomial smoothing of data presented in a white paper by Landauer Inc. ${ }^{[13]}$ The 
best-fit equation for determining an energy conversion factor was ECF $=3 \times 10^{-6} \times 0.67 \mathrm{kVp}^{3}-0.0007 \times 0.67 \mathrm{kVp}^{2}+0.0453$ $\times 0.67 \mathrm{kVp}+0.1005$.

Doses from OSLDs at different positions within a tissue or organ were averaged to express the average tissue-absorbed dose in micrograys $(\mu \mathrm{Gy})$. The products of these values and the percentage of a tissue or organ irradiated in a radiographic examination were used to calculate the equivalent dose $\left(\mathrm{H}_{\mathrm{T}}\right)$ in micro-Sieverts $(\mu \mathrm{Sv})^{[14]}$. The tibia fraction that is irradiated in different field sizes is seen in Table 2.

Table 2. Fraction of tibia irradiated by FOV and patient age.

\begin{tabular}{llll}
\hline Patient Age & $\mathbf{1 0} \mathbf{~ c m}$ FOV Height & $\mathbf{2 0} \mathbf{~ c m}$ FOV Height & Tibia Length [3] \\
\hline 5 & 0.25 & 0.8 & 20 \\
10 & 0.1 & 0.5 & 29 \\
Adult & 0.1 & 0.4 & 36 \\
\hline
\end{tabular}

For bone marrow, the equivalent dose to the whole-body bone marrow was calculated using the summation of the individual equivalent doses to the ankle and foot bones and tibia and fibula. The determination of these equivalent doses was based on the distribution of active bone marrow throughout the body and was calculated for different ages (see Table 3) ${ }^{[15]}$. Active marrow is not typically located in the bones of the foot after the age of 10 or the tibia and fibula after the age of $15^{[16]}$. For bone surface, a correction factor based on experimentally determined mass energy attenuation coefficients for bone and muscle irradiated with mono-energetic photons was applied. An effective beam energy estimated to be $2 / 3$ rds of the peak beam energy for the x-ray unit was used to determine bone /soft tissue attenuation ratios. A linear fit $\left(\mathrm{R}^{2}=0.996\right)$ of ratios from 40 to $80 \mathrm{kV}$ from published data ${ }^{[17]}$ produced the following equation: bone /muscle attenuation ratio $=-0.0618 \times 0.67 \mathrm{kVp}+6.9406$.

Table 3. Percentage of total body active marrow located in foot, ankle, and lower leg bones by age

\begin{tabular}{llll}
\hline Bones $\backslash$ Age (years) & $\mathbf{5}$ & $\mathbf{1 0}$ & $\mathbf{1 8}$ \\
\hline Tibia and Fibula \% active marrow & 9.0 & 5.5 & 0 \\
Ankle and foot $\%$ active marrow & 2.5 & 0 & 0 \\
\hline
\end{tabular}

The proportion of skin surface area in the foot and ankle region directly exposed during CBCT imaging is estimated as 5\% of the total body for a $30 \mathrm{~cm}$ scan volume, $4 \%$ for a $20 \mathrm{~cm}$ scan volume and $3 \%$ for a $10 \mathrm{~cm}$ scan volume to calculate radiation weighted dose to the skin. Similarly, bone surface and muscle exposures are estimated to represent $5 \%, 4 \%$, or $3 \%$ of the total body complement for these tissues for the $30 \mathrm{~cm}, 20 \mathrm{~cm}$, and $10 \mathrm{~cm}$ volumes respectively.

Effective dose (E) is a calculation that permits comparison of the detriment of different exposures to ionizing radiation to an equivalent detriment produced by a full body dose of radiation. $\mathrm{E}$, expressed in $\mu \mathrm{Sv}$, is calculated using the equation: $\mathrm{E}$ $=\sum \mathrm{w}_{\mathrm{T}} \times \mathrm{H}_{\mathrm{T}}$, where $\mathrm{E}$ is the summation of the products of the tissue weighting factor $\left(\mathrm{w}_{\mathrm{T}}\right)$, which represents the relative contribution of that organ or tissue to the overall risk, and the equivalent dose $\mathrm{H}_{\mathrm{T}}{ }^{[14]}$. The whole-body risk is found by the summation of the equivalent doses to all tissues or organs exposed ${ }^{[14]}$.

Tissue weighting factors used in the 2007 ICRP calculation of effective dose include 14 independently weighted tissues and a group of 14 remainder tissues. Because the uterus/cervix is present only in females, and prostate only in males, the number used in the weighted averaging of remainder tissues is 13 . Of remainder tissues, only muscle is directly exposed in foot and ankle imaging. Therefore, remainder dose was calculated from muscle located dosimeters and multiplied by the $\%$ full body distribution ( $3 \%$ to $5 \%$ ), the proportional contribution to remainder weight $(0.08)$, and the remainder weight (0.12). 
The risk of cancer developing years after exposure to ionizing radiation is the principle health related concern associated with diagnostic radiographic imaging. The ICRP has determined that a cancer risk coefficient of $5.5 \times 10^{-2} \mathrm{~Sv}^{-1}$ is consistent with currently available data ${ }^{[14]}$.

\section{Results}

Table 4 demonstrates effective dose calculations for large FOV CBCT examinations by examination protocol and patient age. Higher doses were associated with younger age $(p=0.0185)$ and higher $k \mathrm{Vp}(p=0.0399)$. Comparison of the difference between examinations of each foot independently vs. simultaneous examination of both feet demonstrated a reduction in total dose when both feet are examined together $(p=0.0124)$.

Table 4. PedCAT Large Field of View effective doses in $\mu \mathrm{Sv}$

\begin{tabular}{lllllll}
\hline Foot Protocol & Grid & $\mathbf{k V p}$ & $\mathbf{m A s}$ & $\begin{array}{l}\mathbf{5} \text { year-old } \\
\text { child }\end{array}$ & $\begin{array}{l}\text { 10 year-old } \\
\text { child }\end{array}$ & adult \\
\hline single foot & No & 120 & 4.524 & 28.7 & 13.3 & 3.8 \\
single foot & No & 100 & 4.524 & 17.1 & 8.0 & 2.6 \\
single foot & YES & 120 & 6.786 & 45.4 & 20.9 & 5.8 \\
two feet & No & 120 & 4.524 & 43.2 & 20.7 & 6.4 \\
two feet & No & 100 & 4.524 & 27.9 & 13.1 & 4.3 \\
two feet & YES & 120 & 6.786 & 79.9 & 36.3 & 10.4 \\
\hline difference & No & 120 & 4.524 & 14.2 & 5.9 & 1.2 \\
2 x single foot vs. & No & 100 & 4.524 & 6.2 & 3.0 & 0.9 \\
two feet scan & YES & 120 & 6.786 & 10.8 & 5.6 & 1.3 \\
\hline
\end{tabular}

Table 5 provides effective doses for medium field of view CBCT examinations of a single foot. As with the largest field of view, higher doses were associated with increasing $\mathrm{mAs}$, higher $\mathrm{kVp}$, and younger age. A small reduction in dose is seen when examining the anterior aspect of the foot (toe at edge of field) as opposed to the posterior aspect of the foot (heal at edge of field).

Table 5. PedCAT Medium Field of View effective doses in $\mu \mathrm{Sv}$

\begin{tabular}{lllllll}
\hline Foot Protocol & Grid & kVp & mAs & $\begin{array}{l}\mathbf{5} \text { year-old } \\
\text { child }\end{array}$ & $\begin{array}{l}\text { 10 year-old } \\
\text { child }\end{array}$ & adult \\
\hline single foot & No & 120 & 4.524 & 17.7 & 6.5 & 2.3 \\
single foot & No & 100 & 4.524 & 9.5 & 3.7 & 1.4 \\
single foot & YES & 120 & 6.786 & 29.6 & 10.8 & 3.7 \\
single foot & YES & 120 & 5.655 & 21.7 & 8.0 & 2.7 \\
single foot (front foot) & YES & 120 & 4.524 & 18.3 & 6.6 & 2.2 \\
\hline
\end{tabular}

Table 6 provides effective doses for small field of view CBCT examinations. Again, higher dose is associated with younger age and scanning of the posterior aspect of the foot.

Table 6. PedCAT Small FOV examinations at $120 \mathrm{kVp}, 4.5 \mathrm{~mA}$ : effective doses in $\mu \mathrm{Sv}$

\begin{tabular}{llll}
\hline Foot Protocol & $\mathbf{5}$ year-old child & $\mathbf{1 0}$ year-old child & adult \\
\hline single foot-ankle & 2.9 & 1.2 & 0.9 \\
single foot-toes & 2.1 & 0.8 & 0.5 \\
\hline
\end{tabular}


Table 7 provides effective doses for combined Anterio-Posterior, Lateral, and oblique conventional 2D projections.

Table 7. Anterio-Posterior + Lateral + oblique, $60 \mathrm{kVp}$ conventional 2D projections*

\begin{tabular}{llllll}
\hline \multirow{2}{*}{ Examination } & \multicolumn{5}{l}{ Exposure settings by patient size (mAs) } \\
\cline { 2 - 6 } & child & teen & small adult & medium adult & large adult \\
\hline Foot AP & 1 & 1.6 & 2 & 3.6 & 4.5 \\
Foot Oblique & 1 & 1.6 & 2 & 3.6 & 4.5 \\
Foot Lateral & 1.6 & 2 & 3.6 & 4.5 & 6.3 \\
total mAs & 3.6 & 5.2 & 7.6 & 11.7 & 15.3 \\
\hline \multirow{2}{*}{ Adult } & Effective Dose & & & & \\
10 yo Child & 0.2 & 0.2 & 0.4 & 0.6 & 0.7 \\
5 yo Child & 0.4 & 0.5 & 0.8 & 1.2 & 1.6 \\
\hline
\end{tabular}

${ }^{*}$ Cells highlighted in green represent standard exposure setting for patient age

Table 8 provides effective doses for standard and automatic exposure controlled MDCT examinations.

Table 8. MDCT $20 \mathrm{~cm}$ scan, $120 \mathrm{kVp}, 100 \mathrm{mAs}$ effective doses in $\mu \mathrm{Sv}$

\begin{tabular}{llll}
\hline & $\mathbf{5}$ year-old child & $\mathbf{1 0}$ year-old child & adult \\
\hline MDCT-CARE-DOSE & 181 & 67 & 23 \\
MDCT-Standard protocol & 200 & 74 & 25 \\
\hline
\end{tabular}

Figure 4 provides a graphic comparison of effective doses for child and adult foot/ankle radiography for each of the 2D and 3D examinations evaluated in this study. Doses in decreasing order were Standard MDCT $>$ AEC MDCT $>$ CBCT $>2 D$ radiography $(\mathrm{p}=0.0013)$.

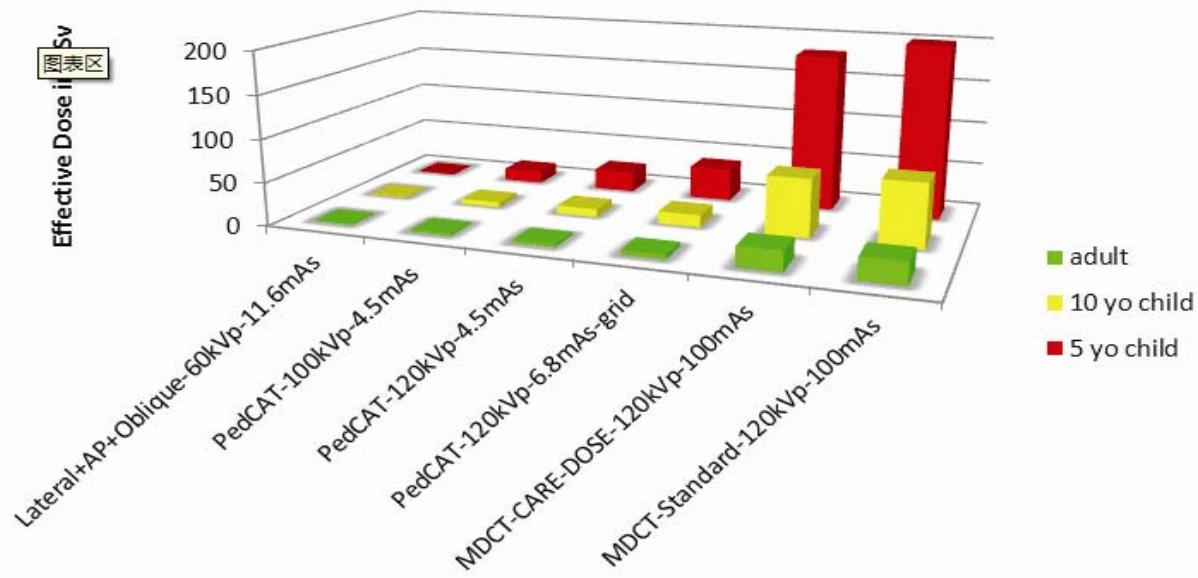

Figure 4. Comparison of effective doses for child and adult foot/ankle radiography 


\section{Discussion}

Although historical concern with the risk of radiographic imaging of the foot dates back to the use of fluoroscopy to fit shoes, little dose and risk information about currently used imaging modalities is available ${ }^{[18]}$.

Doses for children in this study were estimated based on reported differences in marrow percentage and proportion of lower leg exposed during imaging. Because an adult phantom foot was used in this study, actual pediatric doses are likely to be marginally lower due to reduced scattering absorption within a smaller volume of tissue. Automatic exposure control might be expected to further reduce MDCT doses for a smaller foot size. Effective doses for a five-year old patient represent a high dose limit for PedCAT® examinations; however, many five year olds would not meet the height requirement for use of the PedCAT ${ }^{\circledR}$ for foot examination. Means from height and age tables suggest that patients would not routinely meet the PedCAT ${ }^{\circledR}$ height restrictions until the age of eight. The majority of podiatric and foot orthopedic patients will be adults. Therefore, the adult range of effective dose for the PedCAT® examination options will be typical. This ranges from less than a microsievert for a small field examination of the anterior foot, to a high of a little more that 10 $\mu \mathrm{Sv}$ for a two-foot, grid-enhanced examination. By comparison, the standard protocol MDCT scan produced a dose of 25 $\mu \mathrm{Sv}$ while the 3-projection conventional 2D series provided less than $1 \mu \mathrm{Sv}$ of dose. To place these doses in perspective, a standard MDCT scan produced an effective dose comparable to 4 days of per capita background exposure for the average US resident $(8 \mu \mathrm{Sv} /$ day $){ }^{[19]}$. The highest dose, large FOV PedCAT ${ }^{\circledR}$ examination, is just a little more than a single day of background radiation.

\section{Conclusion}

The effective dose for small FOV CBCT examinations is comparable to 1-3 hours of per-capita background dose. Such doses are negligible; therefore, the dose of radiation should not be a concern when considering the use of PedCAT ${ }^{\circledR}$ CBCT imaging for foot/ankle examination.

\section{References}

[1] Judd DB, Kim DH. Foot fractures frequently misdiagnosed as ankle sprains. Am Fam Physician. 2002 Sep; 66(5): 785-94.

[2] Rodop O, Mahiroğulları M, Akyüz M, Sönmez G, Turgut H, Kuşkucu M. Missed talar neck fractures in ankle distortions. Acta Orthop Traumatol Turc. 2010; 44(5): 392-6. http://dx.doi.org/10.3944/AOTT.2010.2328.

[3] Vanhoenacker FM, Bernaerts A, Gielen J, Schepens E, De Schepper AM. Trauma of the pediatric ankle and foot. JBR-BTR. 2002 Aug-Sep; 85(4): 212-8.

[4] Coughlin MJ, Grimes JS, Traughber PD, Jones CP. Comparison of radiographs and CT scans in the prospective evaluation of the fusion of hindfoot arthrodesis. Foot Ankle Int. 2006 Oct; 27(10): 780-7.

[5] Muthukumar T, Butt SH, Cassar-Pullicino VN. Stress fractures and related disorders in foot and ankle: plain films, scintigraphy, CT, and MR Imaging. Semin Musculoskelet Radiol. 2005 Sep; 9(3):210-26. http://dx.doi.org/10.1055/s-2005-921941

[6] Brenner DJ, Hall EJ. Computed tomography--an increasing source of radiation exposure. N Engl J Med. 2007 Nov 29; 357(22): 2277-84. http://dx.doi.org/10.1056/NEJMra072149

[7] Smith-Bindman R, Lipson J, Marcus R, Kim KP, Mahesh M, Gould R, Berrington de González A, Miglioretti DL. Radiation dose associated with common computed tomography examinations and the associated lifetime attributable risk of cancer. Arch Intern Med. 2009 Dec 14; 169(22): 2078-86. http://dx.doi.org/10.1001/archinternmed.2009.427.

[8] Brenner D, Elliston C, Hall E, Berdon W. Estimated risks of radiation-induced fatal cancer from pediatric CT. AJR Am J Roentgenol. 2001 Feb; 176(2): 289-96.

[9] European Commission: Radiation Protection No 172: Cone Beam CT for Dental and Maxillofacial Radiology. Evidence based guidelines. A report prepared by the SEDENTEXCT project. 2012 Available from: http://www.sedentexct.eu/files/radiation_protection_172.pdf

[10] Ludlow JB, Ivanovic M. Comparative dosimetry of dental CBCT devices and 64-slice CT for oral and maxillofacial radiology. Oral Surg Oral Med Oral Pathol Oral Radiol Endod. 2008 Jul; 106(1):106-14. http://dx.doi.org/10.1016/j.tripleo.2008.03.018. Epub 2008 May 27. http://dx.doi.org/10.1016/j.tripleo.2008.03.018 
[11] Zbijewski W, De Jean P, Prakash P, Ding Y, Stayman JW, Packard N, Senn R, Yang D, Yorkston J, Machado A, Carrino JA, Siewerdsen JH. A dedicated cone-beam CT system for musculoskeletal extremities imaging: design, optimization, and initial performance characterization. Med Phys. 2011 Aug; 38(8): 4700-13. http://dx.doi.org/10.1118/1.3611039

[12] Tuominen EKJ, Kankare J, Koskinen SK, Mattila KT. Weight-Bearing CT Imaging of the Lower Extremity. AJR. 2013 Jan; 200(1): 146-8. http://dx.doi.org/10.2214/AJR.12.8481.

[13] Yahnke CJ, Hanify RD, Salasky MR. Microstar Calibration Conversion Factors for DOTs. in: MicroSTAR v2.0 User Manual Available from Landauer Inc: http://www.landauer.com

[14] Valentin J. The 2007 Recommendations of the International Commission on Radiological Protection. Publication 103. Ann ICRP. 2007; 37: 1- 332.

[15] Christy M. Active bone marrow distribution as a function of age in humans. Phys Med Biol. 1981 May; 26(3): 389-400. http://dx.doi.org/10.1088/0031-9155/26/3/003

[16] Scheuer L, Black S. Developmental Juvenile Osteology. Elsevier Academic Press, 2000.

[17] Physical Aspects of Irradiation, NBS Handbook No. 85. Washington DC: US Government Printing Office, 1964 Mar; p.3.

[18] Lewis L, Caplan PE. The shoe-fitting fluoroscope as a radiation hazard. California Medicine 1950; 72: 26-30.

[19] National Council on Radiation Protection and Measurements. Ionizing Radiation Exposure of the Population of the United States (Report No. 160), Bethesda, MD. 2009, The Council. 